\title{
Glycosylceramide modifies the flavor and metabolic characteristics of sake yeast
}

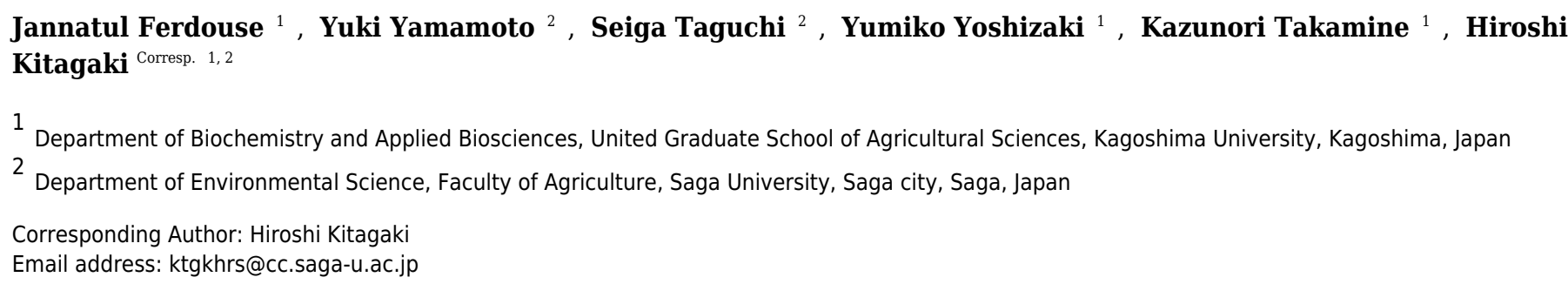

In the manufacture of sake, Japanese traditional rice wine, sake yeast is fermented with koji, which is steamed rice fermented with non-pathogenic fungus Aspergillus oryzae. During fermentation, sake yeast requires lipids, such as unsaturated fatty acids and sterols, in addition to substances provided by koji enzymes for fermentation. However, the role of sphingolipids on the brewing characteristics of sake yeast has not been studied. In this study, we revealed that glycosylceramide, one of the sphingolipids that is abundant in koji, affects yeast fermentation. The addition of soy, $A$. oryzae, and $G$. frondosa glycosylceramide conferred similar effect on the flavor profiles of sake yeast. In particular, the addition of $A$. oryzae and $G$. frondosa glycosylceramide were very similar in terms of the decreases in ethyl caprylate and ethyl 9-decenoate. Addition of soy glycosylceramide induced metabolic changes to sake yeast such as a decrease in glucose, increases in ethanol and glycerol and changes in several amino acids and organic acids concentrations. TCA cycle, pyruvate metabolism, starch and sucrose metabolism, and glycerolipid metabolism were overrepresented in the cultures incubated with sake yeast and soy glycosylceramide. This is the first study of the effect of glycosylceramide on the flavor and metabolic profile of sake yeast. 
1 Glycosylceramide modifies the flavor and metabolic characteristics of sake yeast

2

3 Jannatul Ferdouse ${ }^{1,2}{ }^{\dagger}$, Yuki Yamamoto $^{2 \dagger}$, Seiga Taguchi ${ }^{2}$, Yumiko Yoshizaki ${ }^{1}$, Kazunori

4 Takamine $^{1}$, and Hiroshi Kitagaki ${ }^{1,2 *}$

5

61 Department of Biochemistry and Applied Biosciences, United Graduate School of Agricultural

7 Sciences, Kagoshima University, 1-21-24, Korimoto, Kagoshima 890-8580, Japan

82 Department of Environmental Science, Faculty of Agriculture, Saga University, Saga city, Saga

9 840-8502, Japan

10

11 *Address correspondence to Hiroshi Kitagaki, E-mail: ktgkhrs@,cc.saga-u.ac.jp

$12 \dagger$ These authors equally contributed to this work. 


\section{ABSTRACT}

16 In the manufacture of sake, Japanese traditional rice wine, sake yeast is fermented with koji, which 17 is steamed rice fermented with non-pathogenic fungus Aspergillus oryzae. During fermentation, 18 sake yeast requires lipids, such as unsaturated fatty acids and sterols, in addition to substances 19 provided by koji enzymes for fermentation. However, the role of sphingolipids on the brewing characteristics of sake yeast has not been studied. In this study, we revealed that glycosylceramide, one of the sphingolipids that is abundant in koji, affects yeast fermentation. The addition of soy, A. oryzae, and G. frondosa glycosylceramide conferred similar effect on the flavor profiles of sake yeast. In particular, the addition of A. oryzae and G. frondosa glycosylceramide were very similar in terms of the decreases in ethyl caprylate and ethyl 9-decenoate. Addition of soy glycosylceramide induced metabolic changes to sake yeast such as a decrease in glucose, increases in ethanol and glycerol and changes in several amino acids and organic acids concentrations. TCA cycle, pyruvate metabolism, starch and sucrose metabolism, and glycerolipid metabolism were overrepresented in the cultures incubated with sake yeast and soy glycosylceramide. This is the first study of the effect of glycosylceramide on the flavor and metabolic profile of sake yeast. 


\section{INTRODUCTION}

Japanese traditional rice wine, sake, uses rice as its raw material. Therefore, rice starch is used as a source of carbohydrates, and saccharification of starch is necessary for subsequent fermentation by sake yeast Saccharomyces cerevisiae. As a saccharifier of starch, koji, which is steamed rice fermented with the non-pathogenic fungus Aspergillus oryzae, has been used traditionally in Japan, as a Japanese version of the malt used in beer in western countries. Koji is then mixed with steamed rice and sake yeast, and thus simultaneous saccharification and fermentation occurs (Kitagaki \& Kitamoto, 2013).

Koji contains various enzymes, including glycosidases, proteases, and lipases. The role of these koji enzymes on sake brewing has been studied intensively. Indeed, yeast can synthesize most substances, including saturated fatty acids, from substances provided from rice and degraded by koji enzymes, allowing them to proliferate and ferment. However, in addition to these substances, yeast needs lipids other than saturated fatty acids. Past studies have been limited to unsaturated fatty acids and $\beta$-sitosterol. For example, unsaturated fatty acids have been shown to facilitate fermentation and decrease isoamylacetate production (Ohta \& Hayashida, 1983; Fujii et al., 1997; Mason \& Dufour, 2000). Unsaturated fatty acids, $\beta$-sitosterol, and phospholipids synergistically affect the fusel alcohols content of beer and decrease the content of volatile esters and medium chain-length fatty acids (Taylor, Thurston \& Kirsop, 1979). In addition, grape phytosterol increases the fermentation rate of yeast (Luparia et al., 2004). However, there has been no report on the effect of sphingolipids on yeast fermentation.

Sphingolipids are a class of lipids that contain amide bonds of sphingoid bases and fatty acids. Serine and palmitoyl-CoA are conjugated to form sphinganine, which is reduced to sphingoid bases, such as phytosphingosine, sphingosine, and dihydrosphingosine. Fatty acids are amidelinked to the sphingoid bases to form ceramides. In ceramides, either phosphate or carbohydrates are linked to the hydroxyl bond at the $1^{\text {st }}$ position. Phosphate is ester-linked to the $1^{\text {st }}$ hydroxyl bond of ceramides, and further diester-linked to inositol or choline to form acid complex sphingolipids, such as inositol-phosphoceramide (IPC), mannose-inositol-phosphoceramide (MIPC), and mannose-(inositol phosphate) $)_{2}$-ceramide (M(IP) ${ }_{2} \mathrm{C}$ ) (Dickson et al., 1997) or sphingomyelin (Okazaki, Bell \& Hannun, 1989). Carbohydrates, including glucose, galactose, sialic acid, and/or their conjugates, are acetal-linked to the $1^{\text {st }}$ hydroxyl bond of ceramide to form glycosylceramides. Exceptionally, Saccharomyces cerevisiae lacks a glycosylceramidesynthesizing enzyme (Saito et al., 2006). Sphingolipids form rafts in the lipid bilayer (Simons \& Ikonen, 1997) and also function as signaling lipids (Hannun \& Obeid, 2008).

In previous studies, we revealed that one of the sphingolipids, glycosylceramide, having galactose $(19.2 \%)$ or glucose $(80.8 \%)$ as the monohexosyl moiety, 9-methyl-4,8-sphingadienine as the sphingoid base moiety, and 2'-hydroxyoctadecanoic acid as the fatty acid moiety, is contained in 
69 shochu and sake koji (Hamajima et al., 2016; Hirata et al., 2012). Furthermore, we have revealed that the glycosylceramide contained in shochu koji increased the alkali tolerance and modified the flavor profile of shochu yeast (Sawada et al., 2015). However, the effect of koji glycosylceramide on the fermentation profile of sake yeast remains unknown. Therefore, in the present study, we investigated the effect of koji glycosylceramide on the brewing characteristics of sake yeast.

\section{MATERIALS AND METHODS}

\section{Strains, reagents, and materials}

The $S$. cerevisiae sake yeast (isolated and distributed in 2003 from the Brewing Society of Japan, K7-4) was obtained from the Brewing Society of Japan (Tokyo, Japan). Conidia of $A$. oryzae were obtained from Higuchi Moyashi Co., Ltd. (Osaka, Japan). Analytical grade reagents were used. Glycosylceramides of soy and Grifola frondosa were purchased from Funakoshi Co. Ltd. (Tokyo, Japan), and that of $A$. oryzae was purified from the mycelia of $A$. oryzae.

\section{Culture of $A$. oryzae}

Conidia of $A$. oryzae (10 mg) were dissolved in $1 \mathrm{ml}$ of sterile water, and $100 \mu \mathrm{l}$ of the solution was inoculated onto potato dextrose agar $(0.4 \%$ potato starch, $2 \%$ dextrose, $2 \%$ agar $)$ and incubated at $30{ }^{\circ} \mathrm{C}$ for 3 days. An aliquot of the culture was inoculated into $500 \mathrm{ml}$ of potato dextrose medium $(0.4 \%$ potato starch, $2 \%$ dextrose $)$ and incubated at $30{ }^{\circ} \mathrm{C}$ and $200 \mathrm{rpm}$ for 5 days. Mycelia of $A$. oryzae were washed with sterile water three times, freeze-dried for 2 days, and ground with a mortar.

\section{Lipid extraction}

Dried $A$. oryzae mycelia $(0.12 \mathrm{~g})$ in a glass tube with a screw cap were added with $2 \mathrm{ml}$ of chloroform/methanol $(1: 1 \mathrm{v} / \mathrm{v})$ and vortexed for $1 \mathrm{~min}$. To the solution, $2 \mathrm{ml}$ of $0.8 \mathrm{M} \mathrm{KOH}$ dissolved in methanol was added and incubated at $42{ }^{\circ} \mathrm{C}$ and $160 \mathrm{rpm}$ for $30 \mathrm{~min}$. Subsequently, 5 $\mathrm{ml}$ of chloroform and $2.25 \mathrm{ml}$ of water was vortexed until saponification, and centrifuged at $700 \times$ $g$ for $10 \mathrm{~min}$. The lower organic layer was recovered and evaporated under a vacuum.

\section{Separation and detection of lipids using thin layer chromatography (TLC)}

Separation and detection of lipids were performed basically as previously described (Takahashi et al., 2014).The extracted lipids (500 $\mu 1)$ were dried, dissolved in $50 \mu 1$ of chloroform/methanol (2:1 $\mathrm{v} / \mathrm{v}$ ), and $20 \mu \mathrm{l}$ of the solution was spotted onto a TLC plate (Silica gel 60 plate, Merck Millipore Inc., Darmstadt, Germany). The dried plate was developed with saturated chloroform:methanol:acetic acid:water (20: 3.5: 2.3: 0.7 v/v). Cerebroside (Matreya Inc., Pleasant 
104 Gap, PA, USA) was used as an internal standard. To detect glycosylceramide, $2 \mathrm{mg} / \mathrm{ml}$ of orcinol

105

106

107

\section{Semi-purification of glycosylceramide using column chromatography}

Crude extracts of sphingolipids were evaporated under a vacuum, solubilized in $3 \mathrm{ml}$ of chloroform, and applied to silica gel chromatography (diameter $30 \mathrm{~mm}$, column length $300 \mathrm{~mm}$, Silica Gel 60 70-230 mesh, Nacalai Tesque Inc., Kyoto, Japan). First, $600 \mathrm{ml}$ of chloroform was used as an eluent to remove contaminants. Then, $10 \mathrm{ml}$ of ethyl acetate:methanol (v/v, 9:1) was added 24 times. An aliquot $(800 \mu \mathrm{l})$ of each $10 \mathrm{ml}$ fraction was dried, dissolved in $50 \mu 1$ of chloroform/methanol $(2: 1 \mathrm{v} / \mathrm{v})$, and was spotted onto a TLC plate as described above. An Rf value of 0.4 was adopted as the glycosylceramide fraction, as previously reported (Takakuwa et al., 2005).

\section{Purification of glycosylceramide using high performance liquid chromatography (HPLC)}

Fractions that contained glycosylceramides were evaporated under a vacuum, dissolved in $1.5 \mathrm{ml}$ of chloroform, and injected into a $0.5 \mathrm{ml}$ injection loop. Purification was performed as follows; particle size: $5 \mu \mathrm{m}$, diameter: $4.6 \mathrm{~mm}$, length: $250 \mathrm{~mm}$ (Inertsil SIL 100A, GL Science Inc., Tokyo, Japan), column temperature; $30{ }^{\circ} \mathrm{C}$, buffer A: chloroform, buffer B: $95 \%$ methanol, gradient: 0.01 $\min$ A $100 \%, 60 \min$ A $85 \%, 80 \min$ A $10 \%, 90 \min$ A $10 \%, 90.01 \min$ A $100 \%, 100 \min$ A $100 \%, 100.01 \mathrm{~min}$ STOP; rate: $0.7 \mathrm{ml} / \mathrm{min}$. Eluents at 40-50 min were sampled at $1 \mathrm{~min}$ intervals, and aliquots of the fractions were dried and applied to TLC analysis. The fractions that were confirmed to contain purified glycosylceramide were dried under a vacuum (Supplementary Fig. S1) and dissolved in ethanol $(4 \mu \mathrm{g} / \mu \mathrm{l})$.

\section{Co-culture of sake yeast and glycosylceramide}

Sake yeast was incubated in $3 \mathrm{ml}$ of YPD medium (1\% yeast extract, $2 \%$ bactopeptone, $2 \%$ glucose) at $30{ }^{\circ} \mathrm{C}$ for $24 \mathrm{~h}$. Cells were recovered and washed with sterile water. Koji extract solution $\left(5 \mathrm{ml}\right.$, pregelatinized koji weighing $5 \mathrm{~g}$ was mixed with water and incubated at $55{ }^{\circ} \mathrm{C}$ overnight, and the liquid fraction was recovered) was inoculated with nonidet P-40 (final 0.0015\% $\mathrm{v} / \mathrm{v}$ ), with or without $50 \mu \mathrm{l}$ of $4 \mu \mathrm{g} / \mu \mathrm{l}$ glycosylceramide (either from $A$. oryzae, soy, or G. frondosa) dissolved in ethanol and $10^{6}$ cells $/ \mathrm{ml}$ sake yeast (the final glycosylceramide concentration was 40 $\mu \mathrm{g} / \mathrm{ml})$. Sake yeast is exposed to $40 \mu \mathrm{g} / \mathrm{ml}$ koji glycosylceramide during fermentation; therefore, this concentration was adopted. Sterile liquid paraffin $(1 \mathrm{ml})$ was overlaid onto the medium, and the samples were incubated at $15^{\circ} \mathrm{C}$ for 1 week. The volatile and non-volatile compounds of the culture were analyzed. Flow chart of the experimental procedures is shown in Fig. 1. 
140 Sample collection for metabolites analysis

141 After 1 week of fermentation with or without glycosylceramide, the fermented cultures were 142 centrifuged at $-9{ }^{\circ} \mathrm{C}$ and $3200 \times g$ for $3 \mathrm{~min}$. The supernatant $(5 \mathrm{ml})$ was divided into two plastic 143 tubes $(2.5 \mathrm{ml}$ each $)$ and stored at $4{ }^{\circ} \mathrm{C}$ for volatile compound analysis or at $-27^{\circ} \mathrm{C}$ for non-volatile 144 compounds analysis. The pellet was resuspended in $3 \mathrm{ml}$ of MilliQ water (Millipore Inc., 145 Darmstadt, Germany) and centrifuged again under the same conditions. The pellet was soaked in 146 liquid nitrogen for $3.5 \mathrm{~min}$, freeze-dried for $12 \mathrm{~h}$, and stored at $-80^{\circ} \mathrm{C}$.

147

148

149

150

151

152

153

154

155

156

157

158

159

160

161

162

163

164

165

166

167

168

169

170

171

172

173

174

175

\section{Sample preparation for the analysis of volatile compounds}

Fermented cultures were centrifuged at $4{ }^{\circ} \mathrm{C}$ and $3200 \times \mathrm{g}$ for $3 \mathrm{~min}$, and the supernatant was collected. For target products analysis, $900 \mu \mathrm{l}$ of fermentation broth and $100 \mu \mathrm{l}$ of internal standard mixture (methyl hexanoate at $5 \mathrm{mg} / \mathrm{L}$ and $n$-amyl alcohol at $200 \mathrm{mg} / \mathrm{L}$ ) were placed into a $10-\mathrm{ml}$ glass vial on ice. The vial containing the fermentation broth and an internal standard mixture was sealed with a magnetic cap and subjected to GC/MS analysis, as previously described (Yoshizaki et al., 2010).

\section{GC/MS analysis of volatile compounds}

Aroma components of the fermented cultures were analyzed using headspace gas chromatography mass spectrometry (GC/MS) (GC-2010, GCMS-QP2010; Shimadzu, Kyoto, Japan) equipped with a DB-WAX column (60 m; internal diameter, $0.25 \mathrm{~mm} ; 0.5 \mathrm{~m}$; Agilent Technologies, Palo Alto, CA, USA) as previously described (Yoshizaki et al., 2010). Experiments were performed in triplicate from respective independent cultures.

\section{Sample preparation for extracellular non-volatile compounds}

Sample preparation for extracellular non-volatile compounds was performed as described previously (Kadowaki et al., 2017) with minor modifications. The fermented cultures (100 $\mu 1)$ or freeze-dried cells $(10 \mathrm{mg})$ were mixed with $1 \mathrm{ml}$ of chloroform:methanol:water (2:5:2) for extraction. Ribitol solution $(0.2 \mathrm{mg} / \mathrm{ml}, 60 \mu \mathrm{l})$ was added to the solution and incubated for $30 \mathrm{~min}$ at $30{ }^{\circ} \mathrm{C}$ with shaking at $1500 \mathrm{rpm}$. The supernatant $(900 \mu \mathrm{l})$ was collected by centrifugation $\left(4{ }^{\circ} \mathrm{C}\right.$ $16000 \times g$ for $3 \mathrm{~min}$ ) and $400 \mu 1$ of MilliQ water (Millipore Inc., Darmstadt, Germany) was added, mixed, and centrifuged again under the same conditions. The supernatant ( $800 \mu \mathrm{l})$ was evaporated for $3 \mathrm{~h}$ and freeze-dried for $12 \mathrm{~h}$. Methoxyamine $(20 \mathrm{mg} / \mathrm{ml}$ dissolved in pyridine, $100 \mu \mathrm{l})$ was mixed with the freeze-dried extract and incubated at $30{ }^{\circ} \mathrm{C}$ for $90 \mathrm{~min}$ with shaking at $1500 \mathrm{rpm}$. $N$-methyl- $N$-(trimethylsilyl) trifluoroacetamide (MSTFA) $(50 \mu \mathrm{l})$ was added and again incubated at $37{ }^{\circ} \mathrm{C}$ for $30 \mathrm{~min}$ with shaking at $1500 \mathrm{rpm}$. The solution $(70 \mu \mathrm{l})$ was transferred to a vial and subjected to gas chromatography flame ionization detector (GC/FID) analysis. 
177 GC/FID analysis of non-volatile components. Non-volatile metabolic components, which are produced during fermentation with or without glycosylceramide, were analyzed using a GC/FID (GC-2014, Gas Chromatograph, Shimadzu, Kyoto, Japan) with a CP Sil8CB column $(30 \mathrm{~m} \times 0.25$ $\mathrm{mm} \times 0.25 \mu \mathrm{m}$; Agilent Technologies, Palo Alto, CA, USA). The carrier gas was nitrogen, with a column headspace pressure of $73.9 \mathrm{kPa}$ and a flow rate of $0.97 \mathrm{ml} / \mathrm{min}$. The gas chromatography temperature program was as follows: $60^{\circ} \mathrm{C}$ for $2 \mathrm{~min}$, increased to $320^{\circ} \mathrm{C}$ at $13{ }^{\circ} \mathrm{C} / \mathrm{min}$, and held for $17 \mathrm{~min}$. The split ratios for extracellular metabolites were 10 and 2, respectively. The data were analyzed using GC/FID solution software (Labsolution, Shimadzu, Kyoto, Japan). All metabolite concentrations were normalized using ribitol as an internal control. Experiments were performed from respective independent 7 (control) or 8 (soy glycosylceramide-added) cultures.

\section{Measurement of ethanol concentration}

The ethanol concentrations of the fermented cultures were analyzed using a contact combustion system with an alcohol densitometer (Alcohol Checker YSA-200; Yazaki Meter Co. Ltd., Tokyo, Japan) according to the manufacturer's instructions, and as described previously (Katou et al., 2008).

\section{Statistical analysis}

The statistical significances of differences among the averages of volatile flavors were judged using Student's $t$-test and the false discovery rate. The experimental results were expressed as means \pm standard error of the means. For heatmap analysis and integrated pathway analysis, MetaboAnalyst (Xia et al., 2015) was used. Heatmaps were based on interquantile range data filtering, normalization by sum, clustering with Ward's method, distance measure with Euclidean, standardization by autoscale features and without data scaling.

\section{RESULTS}

First, the flavor compounds of the cultures fermented with sake yeast added with or without glycosylceramides were analyzed (Supplementary Table S1-S6). To obtain information on the specificity of the effect of the chemical structures of glycosylceramide, glycosylceramides from Aspergillus oryzae, soy, and G. frondosa were used. A concentration of $40 \mu \mathrm{g} / \mathrm{ml}$ was adopted, because koji contains approximately $240 \mu \mathrm{g} / \mathrm{g}$ of glycosylceramide (Sawada et al., 2015) and koji is contained at a ratio of 1 to 6 of water and rice in the first step of sake brewing. As a result, addition of $A$. oryzae, soy and $G$. frondosa glycosylceramides showed patterns distinct from vehicle control (ethanol-added culture) (Fig. 2). In particular, A. oryzae and G. frondosa glycosylceramides were clustered in proximity, showing similar decreases in ethyl caprylate and 
212 ethyl 9-decenoate and increases in 2-phenylethyl acetate and phenylethyl alcohol (Table 1). Soy

213 glycosylceramide had a similar effect to A. oryzae in terms of the increase in 2-phenylethyl acetate

214 and decreases in ethyl 9-decenoate, isoamylalcohol and acetoin (Table 2).

Next, since all glycosylceramides showed overall similar trends, the metabolites in the cultures fermented with sake yeast added with soy glycosylceramide were analyzed (Fig. 3 and Table 3). As a result, independent cultures incubated with or without soy glycosylceramide were divided into respective distinct clusters (control culture and soy glycosylceramide-added culture), except CONT_6_007,SOY_1_004 and SOY_3_003 (Fig. 3). Many organic acids and amino acids, such as succinate/glycine, malate, glutamate, valine, methionine, pyruvate and threonine, and glycerol were increased and leucine was decreased in sake yeasts added with soy glycosylceramide. (Table 3). The decrease in glucose (Table 3) and increase in ethanol (Fig. 4) indicated an increased fermentation ability of sake yeast incubated with glycosylceramide, which was consistent with a previous study of shochu yeast (Sawada et al., 2015).

To extract information about the metabolism occurring in the yeast cells incubated with glycosylceramide, the metabolome data of the medium incubated with or without glycosylceramide were analyzed using MetaboAnalyst (Xia et al., 2015). Metabolites (glycerol, succinate/glycine, malic acid, glucose, leucine, valine, glutamate, methionine, pyruvate and threonine), which were significantly different $(p<0.05)$ between medium incubated with or without soy glycosylceramide, were selected and further analyzed. As a result, several metabolisms, such as pyruvate metabolism, the TCA cycle, starch and sucrose metabolism, and glycerolipid metabolism were significantly overrepresented in the medium incubated with glycosylceramide (Fig. 5). This result indicated that extracellularly added glycosylceramide has certain effects on the metabolic pathways described above, although the mechanism remains to be determined.

\section{DISCUSSION}

In this study, we first revealed the effect of glycosylceramides on the metabolic and flavor profiles of sake yeast. Soy, A. oryzae and $G$. frondosa glycosylceramides conferred similar effects on the flavor profiles of sake yeast; addition of $A$. oryzae and $G$. frondosa glycosylceramides showed especially high similarity, providing a specificity of the 9-methyl base of the sphingoid base. Addition of soy glycosylceramide induced significant changes to the concentrations of glucose, ethanol, glycerol and several amino acids and organic acids. Several metabolic pathways were altered in sake yeast incubated with soy glycosylceramide. Considering that the content of glycosylceramide differs among koji samples (Sawada et al., 2015), these results suggested that 
koji exerts its effects on yeast fermentation characteristics through glycosylceramide, and that the effect of koji on sake brewing should be evaluated in terms of the effect of the quantity of glycosylceramide contained in koji.

Alcohol fermentation is performed without oxygen, which is required for synthesis of specific lipids; therefore, it has been documented that unsaturated fatty acids (Fujii et al., 1997; Fujiwara et al., 1998) and sterols (Belviso et al., 2007), which involve molecular oxygen, are required for progression of yeast fermentation. In addition, yeast incubated with or without oxygen (Ishtar Snoek \& Yde Steensma, 2007) or that incubated after diauxic shift (Kitagaki et al., 2009) have different gene expression profiles or yeast incubated without oxygen change their cell wall proteins (Kitagaki, Shimoi \& Itoh, 1997). However, the role of sphingolipids in yeast during fermentation has not been described until our recent study (Sawada et al., 2015).

Sphingolipids have several characteristics that are different from other lipids. For example, they are amide-linked and have long fatty acid lengths (C20-26) (Kitagaki et al., 2007). The mechanism of glycosylceramide's effect on yeast fermentation characteristics seems to be through its increasing effect on the yeast membrane fluidity. Indeed, we have shown in a previous report that glycosylceramide shortens the average fluorescence lifetime of $\tau$ of shochu yeast, as measured by trimethylamine-diphenylhexatriene (Sawada et al., 2015). Consistent with this hypothesis, we found that volatile esters were decreased in sake yeast incubated with glycosylceramide (Fig. 2, Table 1 and Table 2).

The effect of glycosylceramide on the physiology of yeast elucidated in this study suggests its impact on the membrane characteristics. It was reported that the addition of unsaturated fatty acid, which also increases membrane fluidity, decreases ethyl ester flavors (Moonjai et al., 2003; Saerens et al., 2008). It was also reported that a 9-methyl base is essential for membrane fluidity (Singh et al., 2012; Takakuwa et al., 2002). However, the specificity of 9-methyl base-containing sphingolipids was not known. Glycosylceramide containing the 9-methyl-4, 8-sphingadienine base, such as $A$. oryzae and $G$. frondosa glycosylceramide, had different effects in decreasing volatile esters, including ethyl caprylate and ethyl 9-decenoate, compared with those produced by soy glycosylceramide containing a 4, 8-sphingadienine base (Table 1 and Table 2), suggesting that 9-methyl base in the sphingoid base has a strong inhibitory effects on these volatile flavors. However, there are several phenomena that cannot be explained only by the increase in membrane fluidity, such as the increase in glycerol and decrease in leucine. Sphingolipids are signal molecules that stimulate protein phosphatase 2A (Dobrowsky et al., 1993) and form rafts in the lipid bilayer (Pralle et al., 2000); therefore, glycosylceramide might act via these mechanisms. It will be intriguing to investigate the effect of $A$. oryzae glycosylceramide on yeasts, which will form the target of our next study.

The behavior, fate, and effects of koji glycosylceramide during fermentation are now starting to 
284

285

286

287

be elucidated. Koji contains abundant $(0.15-0.25 \mathrm{mg} / \mathrm{ml})$ glycosylceramides (Sawada et al., 2015; Sakamoto et al., 2016). Therefore, sake yeast is exposed to a high concentration of glycosylceramide $(40 \mu \mathrm{g} / \mathrm{ml})$ during fermentation. Koji glycosylceramide is bound to the surface of brewery yeasts, and remains there until the end of fermentation (Sawada et al., 2015). In addition, koji glycosylceramide alters the brewing profiles of sake yeast. These results shed new light on the interaction of koji glycosylceramide and brewery yeasts.

The amount of koji, or the extent of propagation of $A$. oryzae on the surface of koji (haze), has been empirically considered as an important criterion to govern yeast fermentation by brewing technicians in the sake industry. It has been proposed that mycelia of $A$. oryzae increase the complex taste of sake, but the precise mechanism has remained unknown. However, together with the data obtained in this study, it can be hypothesized that the amount of lipids, such as glycosylceramide, unsaturated fatty acids, and sterols, contained in koji, is the key to control yeast fermentation.

Sake yeast cells incubated with glycosylceramide showed effects on pyruvate metabolism, the TCA cycle, starch and sucrose metabolism, and glycerolipid metabolism. Lactosylceramide and ceramide cause dysfunction of the mitochondria (Novgorodov et al., 2016; Law et al., 2017); therefore, it might have stimulated the mitochondrial function of sake yeast. Mitochondrial activity has a significant effect on the fermentation profile of sake yeast (Shiroma et al., 2014; Kitagaki \& Takagi, 2014; Motomura, Horie \& Kitagaki, 2012; Sawada \& Kitagaki, 2016); therefore, the upregulation of mitochondrial function in sake yeast might be responsible for the altered fermentation profile. The mechanism of the effect of glycosylceramide on yeast mitochondria requires further research.

\section{CONCLUSIONS}

In conclusion, we have determined the altered fermentation characteristics of sake yeast in response to glycosylceramide, which will enable interpretation of the effect of koji on the fermentation characteristics of yeast.

\section{ACKNOWLEDGEMENTS}

The authors acknowledge Masafumi Kadowaki, Yuki Fujimaru, Marina Ueda, and Eriko Nakahata for their technical assistance.

\section{REFERENCES}

Belviso S, Bardi L, Bartolini AB, Marzona M. 2007. Lipid nutrition of Saccharomyces cerevisiae in winemaking. Canadian Journal of Microbiology 50(9): 669-674 DOI 10.1139/w04-051. 
Dickson R C, Nagiec EE, Wells GB, Nagiec MM, Lester RL. 1997. Synthesis of mannose(inositol-P)2-ceramide, the major sphingolipid in Saccharomyces cerevisiae, requires the IPT1 (YDR072c) gene. Journal of Biological Chemistry 272(47): 29620-29625 DOI 10.1074/jbc.272.47.29620.

Dobrowsky RT, Kamibayashi C, Mumby MC, Hannun YA. 1993. Ceramide activates heterotrimeric protein phosphatase 2A. Journal of Biological Chemistry 268(21): 15523-30.

Fujii T, Kobayashi O, Yoshimoto H, Furukawa S, Tamai Y. 1997. Effect of aeration and unsaturated fatty acids on expression of the Saccharomyces cerevisiae alcohol acetyltransferase gene. Applied and Environmental Microbioogy 63(3):910-915.

Fujiwara D, Yoshimoto H, Sone H, Harashima S, Tamai Y. 1998. Transcriptional co-regulation of Saccharomyces cerevisiae alcohol acetyltransferase gene, ATF1 and delta-9 fatty acid desaturase gene, OLE1 by unsaturated fatty acids. Yeast14(8):711-721 DOI10.1002/(SICI)1097-0061(19980615)14:8<711::AID-YEA263>3.0.CO;2-8.

Hamajima H, Fujikawa A, Yamashiro M, Ogami T, Kitamura S, Tsubata M, Tan S, Matsunaga H, Sawada K, Kumagai S, Hayashi N, Nagao K, Yanagita T, Oka T, Mitsutake S, Kitagaki H. 2016. Chemical analysis of the sugar moiety of monohexosylceramide contained in koji, Japanese traditional rice fermented with Aspergillus. Fermentation 2(1): 2 DOI 10.3390/fermentation2010002.

Hannun YA, Obeid LM. 2008. Principles of bioactive lipid signalling: lessons from sphingolipids. Nature Review Molecular Cell Biology 9(2):139-150 DOI 10.1038/nrm2329.

Hirata M, Tsuge K, Jayakody L, Urano Y, Sawada K, Inaba S, Nagao K, Kitagaki H. 2012. Structural determination of glucosylceramides in the distillation remnants of shochu, the Japanese traditional liquor, and its production by Aspergillus kawachii. Journal of Agricultural and Food Chemistry 60 (46): 11473-11482 DOI 10.1021/jf303117e.

Ishtar Snoek IS, Yde Steensma H. 2007. Factors involved in anaerobic growth of Saccharomyces cerevisiae. Yeast 24(1):1-10. DOI 10.1002/yea.1430.

Kadowaki M, Fujimaru Y, Taguchi S, Ferdouse J, Sawada K, Kimura Y, Terasawa Y, Agrimi G, Anai T, Noguchi H, Toyoda A, Fujiyama A, Akao T, Kitagaki H. 2017. Chromosomal aneuploidy improves brewing characteristics of sake yeast. Applied and Environmental Microbiology 83(24): e01620-17 DOI 10.1128/AEM.01620-17.

Katou T, Kitagaki H, Akao T, Shimoi H. 2008. Brewing characteristics of haploid strains isolated from sake yeast Kyokai no.7. Yeast 25(11): 799-807 DOI 10.1002/yea.1634. 
Kitagaki H, Cowart LA, Matmati N, Montefusco D, Gandy J, Vaena de Avalos S, Novgorodov S, Zheng J, Obeid LM, Hannun YA. 2009. ISC1-dependent metabolic adaptation reveals an indispensable role for mitochondria in induction of nuclear genes during the diauxic shift in S. cerevisiae. Journal of Biological Chemistry 284(16):10818-10830 DOI 10.1074/jbc.M805029200.

Kitagaki H, Cowart LA, Matmati N, Vaena de Avalos S, Novgorodov SA, Zeidan YH, Bielawski J, Obeid LM, Hannun YA. 2007. Isc1 regulates sphingolipid metabolism in yeast mitochondria. Biochimica et Biophysica Acta

1768(11):2849-2861 DOI 10.1016/j.bbamem.2007.07.019.

Kitagaki H, Kitamoto K. 2013. Breeding research on sake yeast in Japan: history, recent technological advances, and future perspectives. Annual Review of Food Science and Technology 4:215-235 DOI 10.1146/annurev-food-030212-182545.

Kitagaki H, Shimoi H, Itoh K. 1997. Identification and analysis of a static culture-specific cell wall protein, Tir1p/Srp1p in Saccharomyces cerevisiae. European Journal of Biochemistry 249(1): 343-349 DOI 10.1111/j.1432-1033.1997.t01-1-00343.x.

Kitagaki H, Takagi H. 2014. Mitochondrial metabolism and stress response of yeast: Applications in fermentation technologies. Journal of Bioscience and Bioengineering 117(4): 383-393 DOI 10.1016/j.jbiosc.2013.09.011.

Law BA, Liao X, Moore KS, Southard A, Roddy P, Ji R, Sculz Z, Bielawska A, Schulze PC, Cowart LA. 2017. Lipotoxic very-long-chain ceramides cause mitochondrial dysfunction, oxidative stress, and cell death in cardiomyocytes. The FASEB Journal DOI 10.1096/fj.201700300R.

Luparia V, Soubeyrand V, Berges T, Julien,A, Salmon JM. 2004. Assimilation of grape phytosterols by Saccharomyces cerevisiae and their impact on enological fermentations. Applied Microbiology and Biotechnology 65(1): 25-32

DOI 10.1007/s00253-003-1549-3.

Mason AB, Dufour JP. 2000. Alcohol acetyltransferases and the significance of ester synthesis in yeast. Yeast 16(14): 1287-1298 DOI 10.1002/1097-0061(200010)16:14<1287::AID-YEA613>3.0.CO;2-I.

Moonjai N, Verstrepen KJ, Shen HY, Derdelinckx G, Verachtert H, Delvaux FR. 2003. Linoleic acid supplementation of a cropped brewing lager strain: effects on subsequent fermentation performance with serial repitching. Journal of the Institute of Brewing 109(3): 262-272 DOI 10.1002/j.2050-0416.2003.tb00167.x.

Motomura S, Horie K, Kitagaki H. 2012. Mitochondrial activity of sake brewery yeast affects malic and succinic acid production during alcoholic fermentation. Journal of the Institute of Brewing 118(1): 22-26 DOI 10.1002/jib.7. 
Novgorodov SA, Riley CL, Yu J, Keffler JA, Clarke CJ, Van Laer AO, Baicu CF, Zile MR, Gudz TI. 2016. Lactosylceramide contributes to mitochondrial dysfunction in diabetes. Journal of Lipid Research 57(4):546-562 DOI 10.1194/j1r.M060061.

Ohta K, Hayashida S. 1983. Role of tween 80 and monoolein in a lipid-sterol-protein complex, which enhances ethanol tolerance of sake yeasts. Applied and Environmental Microbiology 46(4):821-825.

Okazaki T, Bell RM, Hannun YA. 1989. Sphingomyelin turnover induced by vitamin D3 in HL60 cells. Role in cell differentiation. Journal of Biological Chemistry 264(32):19076-19080.

Pralle A, Keller P, Florin EL, Simons K, Hörber JK. 2000. Sphingolipid-cholesterol rafts diffuse as small entities in the plasma membrane of mammalian cells. Journal of Cell Biology 148(5): 997-1008 DOI 10.1083/jcb.148.5.997.

Saerens SM, Delvaux F, Verstrepen KJ, Van Dijck P, Thevelein JM, Delvaux FR. 2008. Parameters affecting ethyl ester production by Saccharomyces cerevisiae during fermentation. Applied and Environmental Microbiology 74(2):454-461 DOI 10.1128/AEM.01616-07.

Saito K, Takakuwa N, Ohnishi M, Oda Y. 2006. Presence of glucosylceramide in yeast and its relation to alkali tolerance of yeast. Applied Microbiology and Biotechnology 71(4):515-521 DOI 10.1007/s00253-005-0187-3.

Sakamoto M, Sakatani M, Hamajima H, Tsuge K, Nishimukai M, Yanagita T, Nagao K, Mitsutake S, Kitagaki H. 2016. Development of quantitative method for the contents of glycosylceramide contained in Japanese foods brewed with koji and its application. Journal of Brewing Society of Japan 112:1-8 (Japanese).

Sawada K, Kitagaki H. 2016. Residual mitochondrial transmembrane potential decreases unsaturated fatty acid level in sake yeast during alcoholic fermentation. Peer J 4:e1522 DOI 10.7717/peerj.1552.

Sawada K, Sato T, Hamajima H, Jayakody LN, Hirata M, Yamashiro M, Tajima M, Mitsutake S, Nagao K, Tsuge K, Abe F, Hanada K, Kitagaki H. 2015. Glucosylceramide contained in koji mold-cultured cereal confers membrane and flavor modification and stress tolerance to Saccharomyces cerevisiae during co-culture fermentation. Applied and Environmental Microbiology 81(11): 3688-3698

DOI 10.1128/AEM.00454-15.

Shiroma S, Jayakody LN, Horie K, Okamoto K, Kitagaki H. 2014. Enhancement of ethanol fermentation of Saccharomyces cerevisiae sake yeast strain by disrupting mitophagy function. Applied and Environmental Microbiology 80(3): 1002-1012 DOI 10.1128/AEM.03130-13. 
428

429

430

431

432

433

434

435

436

437

438

439

440

441

442

443

444

445

446

447

448

449

450

451

452

Simons K, Ikonen E. 1997. Functional rafts in cell membranes. Nature 387 (6633): $569-572$ DOI 10.1038/42408.

Singh A, Wang H, Silva LC, Na C, Prieto M, Futerman AH, Luberto C, Del Poeta M. 2012. Methylation of glycosylated sphingolipid modulates membrane lipid topography and pathogenicity of Cryptococcus neoformans. Cellular Microbiology 14(4): 500-516 DOI 10.1111/j.1462-5822.2011.01735.x.

Takahashi K, Izumi K, Nakahata E, Hirata M, Sawada K, Tsuge K, Nagao K, Kitagaki H. 2014. Quantitation and structural determination of glucosylceramides contained in sake lees. Journal of Oleo Science 63(1):15 23 DOI 10.5650/jos.ess13086.

Takakuwa N, Saito K, Ohnishi M, Oda Y. 2005. Determination of glucosylceramide contents in crop tissues and by-products from their processing. Bioresour Technol. 96(9):1089-92.

Takakuwa N, Tanji M, Oda Y, Ohnishi M 2002. Distribution of 9-methyl sphingoid base in mushrooms and its effects on the fluidity of phospholipid liposomes. Journal of Oleo Science, 51(11):741-747.

Taylor GT, Thurston PA, Kirsop BH. 1979. The influence of lipids derived from malt spent grains on yeast metabolism and fermentation. Journal of the Institute of Brewing 85:219-227 DOI 10.1002/j.2050-0416.1979.tb03911.x.

Xia J, Sinelnikov IV, Han B, Wishart DS. 2015. MetaboAnalyst 3.0--making metabolomics more meaningful. Nucleic Acids Research 43(W1):251-257 DOI 10.1093/nar/gkv380.

Yoshizaki Y, Yamato H, Takamine K, Tamaki H, Ito K, Sameshima YJ. 2010. Analysis of volatile compounds in shochu koji, sake koji, and steamed rice by gas chromatography-mass spectrometry. Journal of the Institute of Brewing 116(1): 49-55 DOI 10.1002/j.20500416.2010.tb00397.x. 


\section{Table $\mathbf{1}$ (on next page)}

Volatile compounds in the culture of sake yeast added with or without glycosylceramide of $A$. oryzae and $G$. frondosa.

Sake yeasts were incubated in synthetic medium with or without $40 \mu \mathrm{g} / \mathrm{ml}$ glycosylceramide of $A$. oryzae and $G$. frondosa and nonidet P-40 (final $0.0015 \% \mathrm{v} / \mathrm{v}$ ) at $15{ }^{\circ} \mathrm{C}$ for 1 week. Volatile compounds were analysed using headspace gas chromatography mass spectrometry (GC/MS). GlcCer indicates glycosylceramide. The results are the mean values with standard errors of triplicate independent experiments. The relative percentage as compared to the mean value of the control culture is shown. Volatile compounds which were significantly different between control and GlcCer-added culture as judged by false discovery rate $(p<$ 0.05 , indicated by $*$ ) are described. $P$ values were calculated using unpaired one-tailed Student's t-test. Further details are described in Materials and Methods. 
1 Table 1. Volatile compounds in the culture of sake yeast added with or without 2 glycosylceramide of $A$. oryzae and $G$. frondosa

3

\begin{tabular}{|c|c|c|c|c|c|}
\hline \multirow[b]{2}{*}{$\begin{array}{c}\text { Volatile } \\
\text { Compounds }\end{array}$} & \multirow{2}{*}{$\begin{array}{c}\text { Control } \\
\text { Relative } \\
\text { percentage } \\
(\%)\end{array}$} & \multicolumn{2}{|c|}{ GlcCer of $A$. oryzae } & \multicolumn{2}{|c|}{ GlcCer of $G$. frondosa } \\
\hline & & $\begin{array}{c}\text { Relative } \\
\text { percentage } \\
(\%)\end{array}$ & $p$ value & $\begin{array}{c}\text { Relative } \\
\text { percentage } \\
(\%)\end{array}$ & $p$ value \\
\hline $\begin{array}{c}\text { Ethyl } \\
\text { Caprylate }\end{array}$ & $100.0 \pm 13.1$ & $\begin{array}{c}56.4 \pm 1.7 \\
8\end{array}$ & $0.0272 *$ & $\begin{array}{c}50.4 \pm 1.0 \\
7\end{array}$ & $0.0185^{*}$ \\
\hline $\begin{array}{c}\text { Ethyl 9- } \\
\text { Decenoate }\end{array}$ & $100.0 \pm 20.9$ & $\begin{array}{c}38.4 \pm 5.4 \\
9\end{array}$ & $0.0401 *$ & $\begin{array}{c}26.2 \pm 2.3 \\
5\end{array}$ & $0.0227^{*}$ \\
\hline $\begin{array}{c}2- \\
\text { Phenylethyl } \\
\text { Acetate }\end{array}$ & $100.0 \pm 19.6$ & $351 \pm 34.6$ & $0.00338 *$ & $431 \pm 27.7$ & $0.000671 *$ \\
\hline $\begin{array}{c}\text { Phenylethyl } \\
\text { Alcohol }\end{array}$ & $100 \pm 3.59$ & $159 \pm 19.3$ & $0.0342 *$ & $216 \pm 9.56$ & $0.000376^{*}$ \\
\hline
\end{tabular}

4 


\section{Table 2 (on next page)}

Volatile compounds in the culture of sake yeast added with or without glycosylceramide of $A$. oryzae and soy.

Sake yeasts were incubated in synthetic medium with or without $40 \mu \mathrm{g} / \mathrm{ml}$ glycosylceramide of $A$. oryzae and soy and nonidet P-40 (final $0.0015 \% \mathrm{v} / \mathrm{v}$ ) at $15{ }^{\circ} \mathrm{C}$ for 1 week. Volatile compounds were analysed using headspace gas chromatography mass spectrometry (GC/MS). GlcCer indicates glycosylceramide. The results are the mean values with standard errors of triplicate independent experiments. The relative percentage as compared to the mean value of the control culture is shown. Volatile compounds which were significantly different between control and GlcCer-added culture as judged by false discovery rate $(p<$ 0.05 , indicated by $*$ ) are described. $P$ values were calculated using unpaired one-tailed Student's t-test. Further details are described in Materials and Methods. 
1

2 Table 2. Volatile compounds in the culture of sake yeast added with or without 3 glycosylceramide of $A$. oryzae and soy

4

5

\begin{tabular}{|c|c|c|c|c|c|}
\hline \multirow[b]{2}{*}{$\begin{array}{c}\text { Volatile } \\
\text { Compounds }\end{array}$} & \multirow{2}{*}{\begin{tabular}{|c|} 
Control \\
Relative \\
percentage $(\%)$
\end{tabular}} & \multicolumn{2}{|c|}{ GlcCer of $A$. oryzae } & \multicolumn{2}{|c|}{ GlcCer of soy } \\
\hline & & $\begin{array}{c}\text { Relative } \\
\text { percentage (\%) }\end{array}$ & $p$ value & $\begin{array}{c}\text { Relative } \\
\text { percentage } \\
(\%)\end{array}$ & $p$ value \\
\hline $\begin{array}{l}\text { Isoamyl } \\
\text { Alcohol }\end{array}$ & $100 \pm 4.16$ & $75.1 \pm 5.18$ & $0.0189 *$ & $97.6 \pm 1.93$ & 0.347 \\
\hline Acetoin & $100 \pm 7.51$ & $44.7 \pm 8.22$ & $0.00772 *$ & $84.7 \pm 8.58$ & 0.168 \\
\hline $\begin{array}{l}\text { Ethyl 9- } \\
\text { Decenoate }\end{array}$ & $100 \pm 13.4$ & $35.2 \pm 2.12$ & $0.00886^{*}$ & $61.5 \pm 3.23$ & $0.0427 *$ \\
\hline $\begin{array}{l}\text { 2-Phenylethyl } \\
\text { Acetate }\end{array}$ & $100 \pm 12.9$ & $125 \pm 24.3$ & 0.249 & $192 \pm 18.9$ & $0.0150^{*}$ \\
\hline $\begin{array}{l}\text { Phenylethyl } \\
\text { Alcohol }\end{array}$ & $100 \pm 5.00$ & $90.2 \pm 14.0$ & 0.310 & $143 \pm 10.6$ & $0.0200 *$ \\
\hline
\end{tabular}

6 


\section{Table 3 (on next page)}

Metabolite concentrations in the culture of sake yeast added with or without soy glycosylceramide.

Sake yeasts were incubated in synthetic medium with or without $40 \mu \mathrm{g} / \mathrm{ml}$ soy glycosylceramide and nonidet P-40 (final $0.0015 \% \mathrm{v} / \mathrm{v}$ ) at $15{ }^{\circ} \mathrm{C}$ for 1 week. Metabolites derivatized with methoxyamine and MSTFA, analysed using gas chromatography flame ionization detector (GC/FID) and normalized using ribitol. GlcCer indicates glycosylceramide. The results are the mean values with standard errors of 7 (control) or 8 (GlcCer of soy) independent experiments. The relative percentage as compared to the mean value of the control culture is shown. $P$ values were calculated using unpaired one-tailed Student's $t$-test. Further details are described in Materials and Methods. 
1

2 Table 3. Metabolite concentrations in the culture of sake yeast added with or without soy

3 glycosylceramide

4

5

\begin{tabular}{|c|c|c|c|}
\hline \multirow{2}{*}{$\begin{array}{c}\text { Metabolic } \\
\text { compounds }\end{array}$} & Control & \multicolumn{2}{|c|}{ GlcCer of soy } \\
\cline { 2 - 4 } & $\begin{array}{c}\text { Relative percentage } \\
(\%)\end{array}$ & $\begin{array}{c}\text { Relative percentage } \\
(\%)\end{array}$ & $p$ value \\
\hline Glycerol & $100 \pm 2.55$ & $110 \pm 1.14$ & 0.00140 \\
\hline Succinate/Glycine & $100 \pm 4.05$ & $116 \pm 2.68$ & 0.00376 \\
\hline Malate & $100 \pm 2.54$ & $116 \pm 3.55$ & 0.00191 \\
\hline Glucose & $100 \pm 5.17$ & $71.7 \pm 3.05$ & 0.000513 \\
\hline Glutamate & $100 \pm 0.501$ & $101 \pm 0.315$ & 0.0392 \\
\hline Leucine & $100 \pm 2.73$ & $89.8 \pm 1.86$ & 0.00770 \\
\hline Valine & $100 \pm 5.12$ & $128 \pm 4.62$ & 0.00225 \\
\hline Methionine & $100 \pm 7.23$ & $127 \pm 6.12$ & 0.0130 \\
\hline Pyruvate & $100 \pm 15.5$ & $222 \pm 28.3$ & 0.00235 \\
\hline Threonine & $100 \pm 13.8$ & $146 \pm 9.62$ & 0.0125 \\
\hline
\end{tabular}

6

7

8

9 
Figure 1

Flow chart of the experimental procedures.

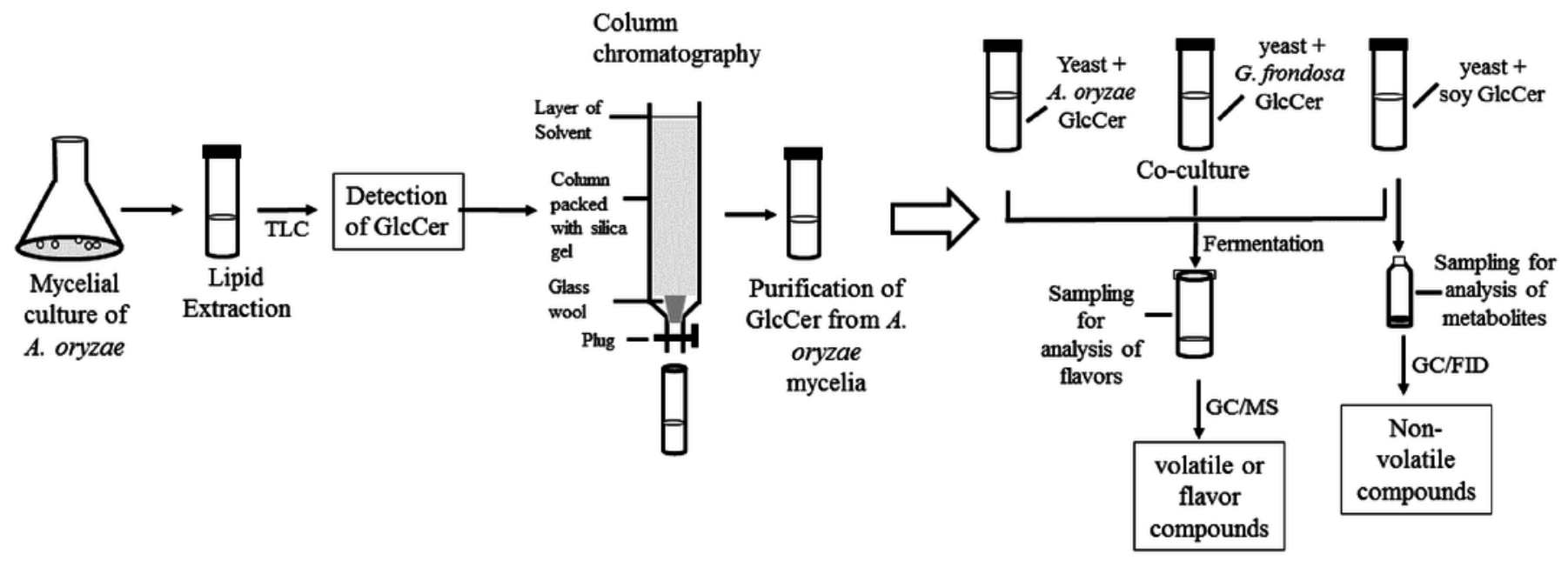




\section{Figure 2}

Heatmap of volatile compounds in the culture of sake yeast incubated with or without glycosylceramide.

Sake yeasts were incubated in synthetic medium containing $40 \mu \mathrm{g} / \mathrm{ml} \mathrm{A}$. oryzae (A1 and A2), soy (S), G. frondosa (G) glycosylceramide or their vehicle control ethanol (E1 and E2) and nonidet P-40 (final concentration $0.0015 \% \mathrm{v} / \mathrm{v}$ ) at $15{ }^{\circ} \mathrm{C}$ for 1 week. Volatile compounds were analyzed using headspace gas chromatography mass spectrometry (GC/MS). A heatmap of volatile compounds was created with Metaboanalyst. 


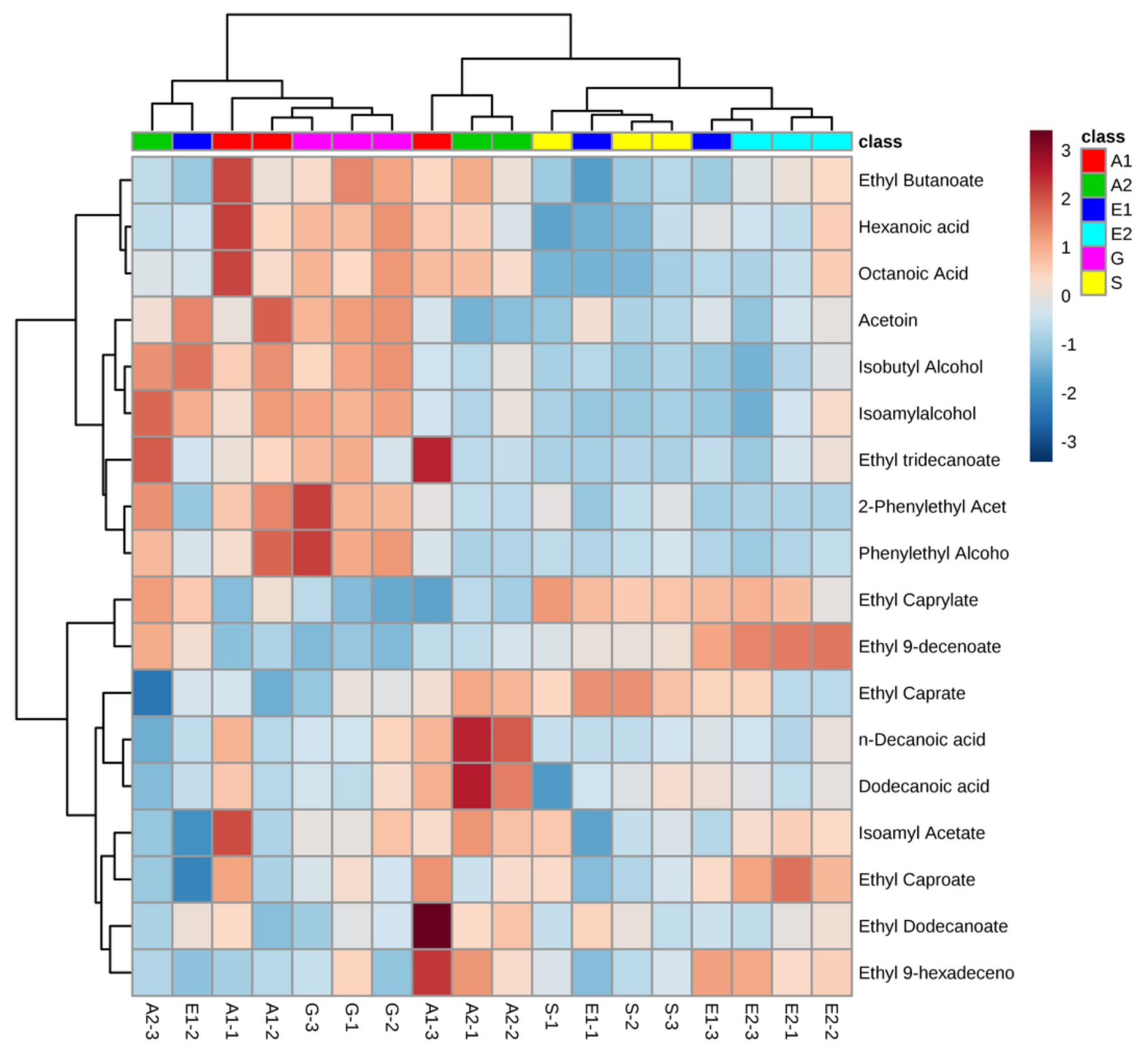




\section{Figure 3}

Heatmap of extracellular metabolite concentrations in the culture of yeast incubated with or without soy glycosylceramide.

Sake yeasts were incubated in synthetic medium containing $40 \mu \mathrm{g} / \mathrm{ml}$ soy glycosylceramide and nonidet P-40 (final concentration $0.0015 \% \mathrm{v} / \mathrm{v}$ ) at $15{ }^{\circ} \mathrm{C}$ for 1 week. Metabolites of the cultures were derivatized with methoxyamine and MSTFA, analysed using GC-FID and normalized using ribitol. A heatmap of metabolites was created with Metaboanalyst. 


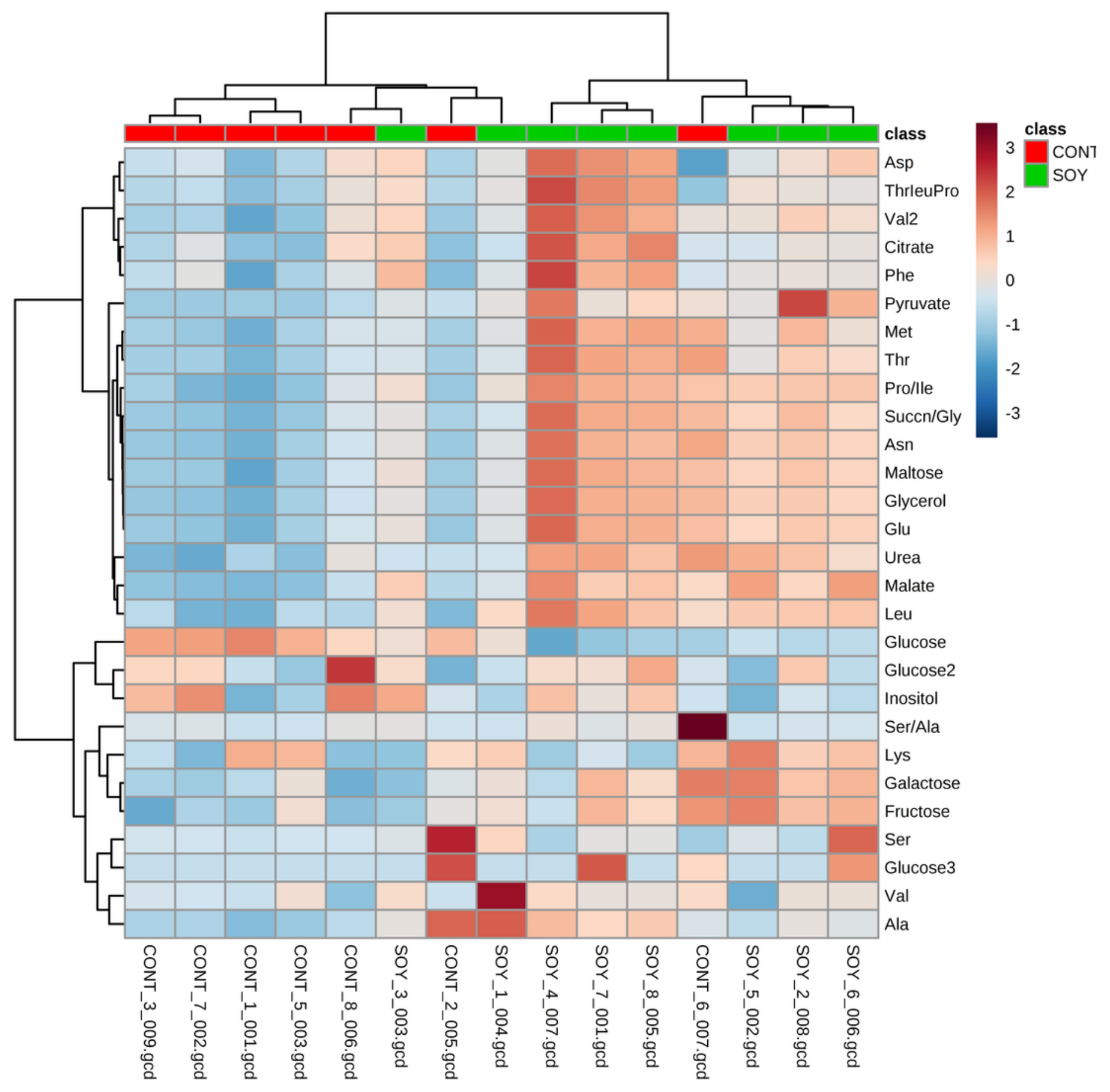




\section{Figure 4}

Ethanol concentrations (\%(vol/vol)) of the culture of sake yeast added with or without soy glycosylceramide.

Sake yeasts were incubated in synthetic medium with or without $40 \mu \mathrm{g} / \mathrm{ml}$ soy glycosylceramide and nonidet P-40 (final $0.0015 \% \mathrm{v} / \mathrm{v}$ ) at $15{ }^{\circ} \mathrm{C}$ for 1 week. The ethanol concentration of fermented culture was analyzed using a contact combustion system with an alcohol densitometer. The results are the mean values with standard errors of triplicate independent experiments. The statistical significance of the difference between the averages was assessed using the unpaired one-tailed Student's $t$-test $(* * *, p<0.001)$.

Ethanol

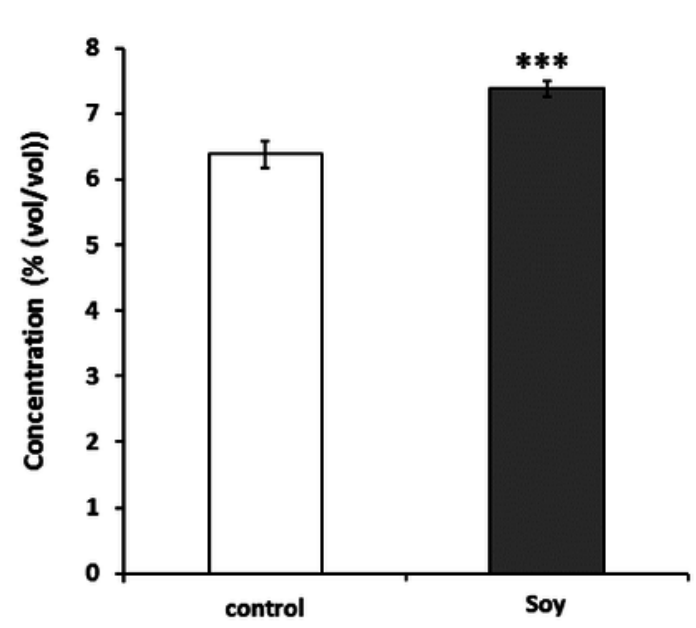




\section{Figure 5}

Pathway analysis of extracellular metabolites of sake yeast incubated with or without soy glycosylceramide.

The normalized values of metabolites (glycerol, succinate/glycine, malic acid, glucose, leucine, glutamate, valine, methionine, pyruvate, and threonine), which were significantly different $(p<0.05)$ between medium incubated with and without soy glycosylceramide, were used as independent variables for Metaboanalyst.

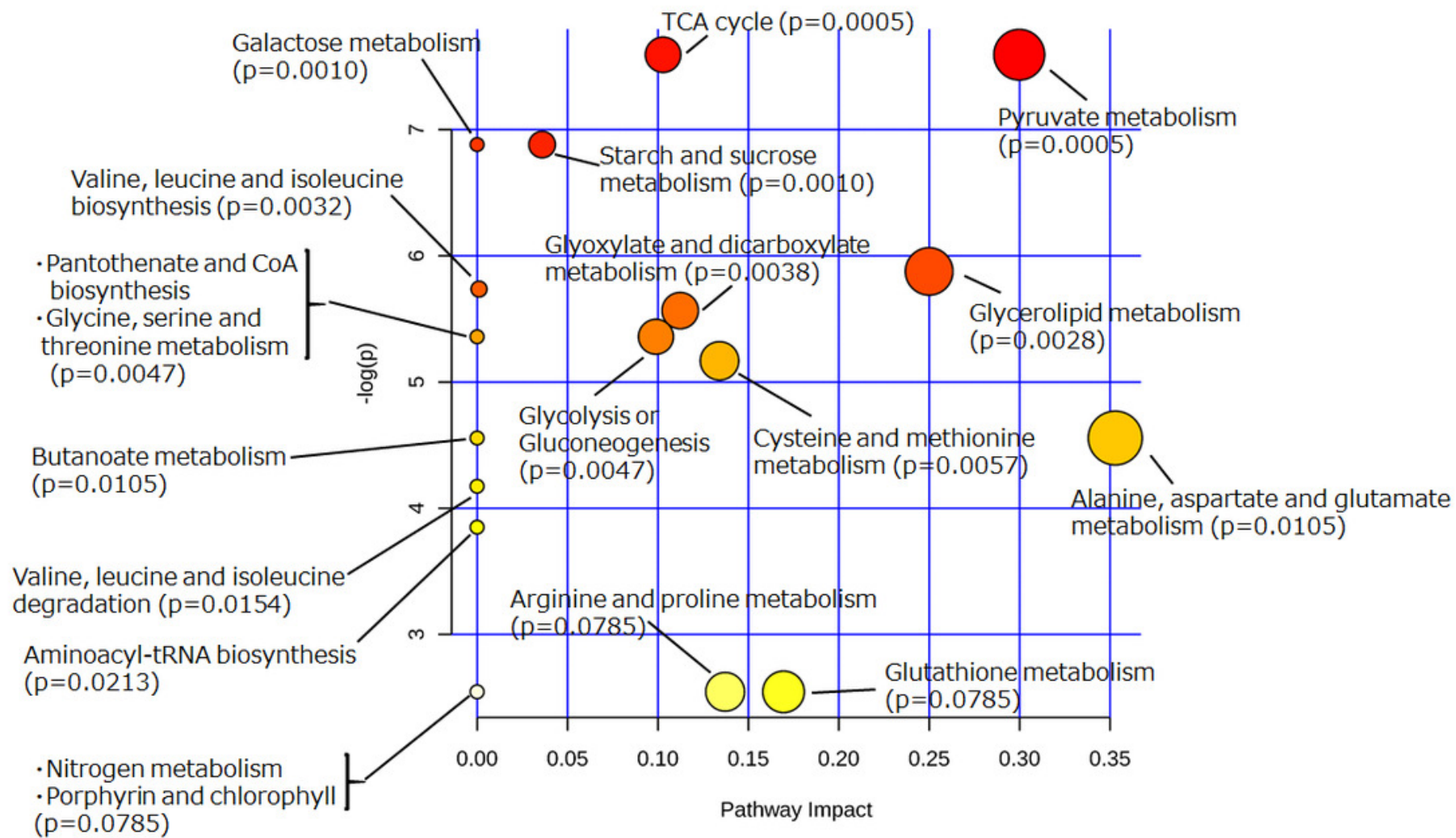

